\section{PROPOSTAS \\ METODOLÓGICAS \\ METHODS GUIDELINES}

\title{
Estudos de Microcusteio aplicados a avaliações econômicas em saúde: uma proposta metodológica para o Brasil
}

\author{
Microcosting methods applied to health economic \\ assessment: a Brazilian methods guideline
}

Ana Paula Beck da Silva Etges 1,2, Rosane Paixão Schlatter ${ }^{1,3}$, Jeruza Lavanholi Neyeloff',3, Denizar Vianna Araújo 1,4, Luciana R. Bahia 1,4, Luciane Cruz ${ }^{1,5}$, Márcia Regina Godoy6, Otávio Neves da Silva Bittencourt ${ }^{7}$, Prisciane Raupp da Rosa ${ }^{5}$, Carisi Anne Polanczyk 1,2,5

DOI: 10.21115/JBES.v11.n1.p87-95

\begin{abstract}
Palavras-chave:
microcusteio, diretriz

metodológica, custos de saúde, métodos de custeio, avaliações de tecnologia em saúde
\end{abstract}

\section{Keywords:}

microcosting, methods guideline, health costs, cost methods, health technology assessment

\begin{abstract}
RESUMO
Objetivo: No cenário da avaliação de tecnologias em saúde (ATS), as estimativas de custos são um fator crítico no desenvolvimento das avaliações econômicas completas, especialmente pelo uso de diferentes metodologias de custeio. A fim de contribuir com a acurácia dos dados de custos usados nessas análises, este artigo sugere recomendações para apuração de custos em saúde no Brasil. Métodos: Reuniram-se pesquisadores de ATS de diferentes expertises e centros de pesquisa do Brasil, e ao longo de dois anos foram conduzidas revisões da literatura nacional e internacional e discussões sobre as formas de abordar a temática. Três simpósios foram realizados reunindo os pesquisadores com o propósito de alcançar o consenso entre os autores sobre as melhores recomendações para a realização de estudos de Microcusteio. Resultados: Consolidou-se em forma de uma recomendação este artigo que representa uma versão compacta da diretriz completa a ser publicada pela Rede Brasileira de Avaliação de Tecnologias em Saúde. A metodologia de Microcusteio é considerada como padrão-ouro para a identificação dos custos em saúde. Os métodos de definição do estudo, coleta e análise de dados apresentados são descritos de modo a permitir uma valoração dos custos validada e homogênea, principalmente para o uso dessa informação em avaliações econômicas de saúde. Conclusão: Essa recomendação tem o propósito de aumentar a acurácia das estimativas dos custos de saúde no nosso meio e homogeneizar a comunicação entre estudos conduzidos por diferentes grupos de pesquisa. Por fim, é esperado que a utilização dessas recomendações contribua para que as decisões baseadas em dados econômicos sejam mais acuradas e equânimes quando da incorporação de tecnologias no país.
\end{abstract}

\begin{abstract}
Objective: In the context of health technology assessment (HTA), cost estimates are a critical factor in the development of economic evaluations, especially through the use of different costing methodologies. In order to contribute to the accuracy of the cost data used in these analyzes, this article suggests recommendations to develop health cost analysis in Brazil. Methods: HTA researchers
\end{abstract}

Recebido em: 06/03/2019. Aprovado para publicação em: 15/03/2019

1. Instituto Nacional de Ciência e Tecnologia de Avaliação em Tecnologias em Saúde (INCT/IATS), Conselho Nacional de Desenvolvimento Científico e Tecnológico (CNPq), Porto Alegre, RS, Brasil.

2. Escola Politécnica da Pontifícia Universidade Católica do Rio Grande do Sul, Porto Alegre, RS, Brasil.

3. Programa de Pós-graduação em Pesquisa Clínica do Hospital de Clínicas de Porto Alegre, Programa de Pós-graduação em

Ensino em Saúde da Universidade Federal do Rio Grande do Sul, Porto Alegre, RS, Brasil.

4. Departamento de Medicina Interna, Universidade do Estado do Rio de Janeiro, Rio de Janeiro, RJ, Brasil.

5. Hospital Moinhos de Vento, Porto Alegre, RS, Brasil.

6. Faculdade de Ciências Econômicas da Universidade Federal de Rio Grande, Rio Grande, RS, Brasil.

7. Universidade Federal de Ciências da Saúde de Porto Alegre, Porto Alegre, RS, Brasil.

Instituição que desenvolveu o estudo: Instituto Nacional de Ciência e Tecnologia de Avaliação em Tecnologias em Saúde (INCT/IATS) no contexto de um projeto inserido no Programa de Apoio ao Desenvolvimento Institucional do SUS (PROADI-SUS) coordenado pelo Hospital Moinhos de Vento em Porto Alegre.

Fomento para o estudo: Este estudo recebeu auxílio financeiro do Programa de Apoio ao Desenvolvimento Institucional do SUS (PROADI-SUS), coordenado pelo Hospital Moinhos de Vento em Porto Alegre.

Autor correspondente: Carisi Anne Polanczyk. Instituto Nacional de Ciência e Tecnologia de Avaliação em Tecnologias em Saúde (INCT/IATS). Universidade Federal do Rio Grande do Sul (UFRGS). Ramiro Barcelos, 2350, prédio 21-507, Porto Alegre, RS, Brasil. CEP: 90035-903. E-mail: cpolanczyk@hcpa.edu.br 
with heterogeneous background and from different Brazilian research centers were engaged on the development of this health cost analysis recommendation over two years. Reviews of national and international literature and discussions on how to approach the theme were conducted. Three symposia were held bringing together the researchers with the purpose of reaching consensus among the authors on the best recommendations for micro-accounting studies. Results: This article was consolidated as a recommendation, which represents a compact version of the complete guideline that will be published by the Brazilian Health Technology Assessment Network (REBRATS). The Microcosting methodology is considered as a gold standard for the analysis of health costs. Methods to define the study, to perform data collection and analysis are described in order to allow a validated and homogeneous cost evaluation, mainly for the use of this information in economic health assessments. Conclusion: This recommendation is intended to increase the health cost estimated accuracy in our country and to homogenize the communication between studies conducted by different research groups. Finally, it is expected that the use of these recommendations will contribute to make decisions based on economic data more accurate and equitable when incorporating health technologies in the country.

\section{Introdução}

Devido ao impacto crescente dos custos associados à absorção de tecnologias em saúde e ao aumento da demanda dos usuários pelos serviços de assistência à saúde, cabe ao gestor público a responsabilidade pela utilização racional dos recursos de forma a atender os direitos e o bem-estar social da coletividade (Piola \& Vianna, 2009). Como instrumento de apoio, a área de avaliação de tecnologias em saúde (ATS) subsidia o gestor na tomada de decisão com base em evidências científicas abrangendo questões clínicas, éticas, legais e de acessibilidade (Brasil, 2008).

Países como Austrália, Canadá, Inglaterra, entre outros, implantaram diretrizes próprias para ATS que incluem a avaliação econômica como requisito básico para análise pelo órgão regulador que recomendará sua inserção ou não no sistema (Brasil, 2008). No Brasil, a partir de 2006, o Ministério da Saúde (MS) definiu a política de incorporação de novas tecnologias no âmbito do Sistema Único de Saúde (SUS), preconizando a avaliação econômica completa como item fundamental para a análise da incorporação de novas tecnologias. Em 2011, por meio da Lei no 12.401, foi referendada a atuação da Comissão Nacional de Incorporação de Tecnologias no SUS, que dispõe sobre a assistência terapêutica e a incorporação de tecnologia em saúde no âmbito do sistema (Brasil, 2011). Em 2012, foi publicada a Diretriz Metodológica para Avaliação Econômica de Tecnologias em Saúde (Brasil, 2012), com a finalidade de padronizar as avaliações realizadas por pesquisadores e técnicos do Ministério, permitindo a possibilidade de revisão técnica e reprodução dos estudos realizados no país. Essas iniciativas estão todas alinhadas à atual Política Nacional de Gestão de Tecnologias em Saúde, cujo objetivo é maximizar os benefícios de saúde a serem obtidos com os recursos disponíveis, assegurando o acesso da população a tecnologias efetivas e seguras, em condições de equidade.

Com o crescimento dos estudos de ATS, a realização das estimativas de custos em saúde passou a ser um fator limitan- te no desenvolvimento das avaliações econômicas, em virtude do uso de diferentes metodologias de custeio e da ausência de sistema de custos informatizados nas instituições públicas, o que dificulta a comparabilidade entre diferentes aferições de custos e entre serviços de saúde (Kolaczinski \& Hanson, 2006; Tan, 2009). A recomendação de metodologias de custeio busca uma homogeneização da aferição de custos por distintos serviços de saúde com adesão a um mesmo padrão, de modo a assegurar que as estimativas de custo resultantes possam ser atribuídas efetivamente ao cuidado de saúde em estudo, sem que diferenças observadas sejam decorrentes de diferenças de aferição ou de falhas na metodologia de apuração de custos (Drummond et al., 2005).

Nesse contexto, este artigo tem como objetivo estabelecer recomendações para a realização de estimativas de custos em saúde utilizando a metodologia de Microcusteio, considerada como padrão-ouro para a identificação dos custos em saúde.

\section{Métodos de construção da diretriz}

Dez pesquisadores de ATS de múltiplas expertises, médicos epidemiologistas, economistas, engenheiros e contadores, de centros de pesquisa do Brasil foram convidados a participar da discussão conceitual referente a essa recomendação. Os pesquisadores foram selecionados em função de suas publicações científicas e atuação em projetos de pesquisas em território nacional sobre o tema custos em saúde.

Ao longo de dois anos, foram conduzidas revisões da literatura nacional e internacional e discussões sobre ferramentas e métodos de avaliação de custos em saúde. Para que isso fosse possível, além de reuniões periódicas por videoconferência, três simpósios foram realizados reunindo o grupo envolvido e pesquisadores internacionais do tema. Esses momentos presenciais permitiram alcançar o consenso entre os autores sobre as melhores recomendações para a realização de estudos de Microcusteio e alinhar estas às recomendações já utilizadas em países como Argentina e Inglaterra.

A diretriz completa, incluindo exemplos e anexos, foi entregue ao MS do Brasil e será publicada pela Rede de Avaliações 
de Tecnologias em Saúde. Este artigo consolida as recomendações essenciais estabelecidas na diretriz.

\section{Métodos de custeio em saúde}

A avaliação dos custos individuais em saúde tem como objetivo auxiliar o tomador de decisão a compreender se as diferenças de custo entre as opções de tratamento são reflexos de variações de custos unitários ou da variação na intensidade da utilização dos recursos e a forma de distribuição dos dados de custo em que se baseiam as estimativas (Tan, 2009). Os métodos de cálculo de custos em saúde possuem como principal característica a forma como são identificados e avaliados os componentes de custos. Em relação à identificação, os custos podem ser estimados por meio dos métodos de macrocusteio ou custeio bruto (gross-costing) ou de Microcusteio (microcosting). E quanto à avaliação dos componentes de custos, os métodos podem ser realizados de cima para baixo (top-down) ou de baixo para cima (bottom-up) (Hrifach et al., 2016; Tan, 2009).

Em conjunto, os itens acima constituem uma matriz de quatro dimensões, que informa sobre o nível de precisão da identificação e a avaliação dos componentes de custos. Temos, assim, os quatro métodos referenciados na literatura para as avaliações econômicas: custeio bruto de cima para baixo, custeio bruto de baixo para cima, Microcusteio de cima para baixo e Microcusteio de baixo para cima (Tan, 2009).

Estudos de custeio bruto trabalham com dados dos componentes de custos em nível agregado, em geral, partindo de bases eletrônicas de dados (Drummond et al., 2005; Hrifach et al., 2016). No custeio bruto, identificam-se somente os componentes de custo que terão impacto no serviço de saúde que está sendo analisado, por exemplo, diárias de internação (Tan, 2009). A abordagem de cima para baixo estima o custo médio por paciente, enquanto a de baixo para cima, o custo individual. A principal desvantagem do custeio bruto para uso em avaliações econômicas é a baixa precisão, uma vez que não possibilita o rastreamento dos custos diretamente ao paciente. É considerado um método pobre em avaliações econômicas, principalmente quando as diárias de internação são o único componente de custo, uma vez que o diagnóstico do paciente tem efeito importante sobre a utilização de recursos (Tan, 2009).

Diversos autores consideram o Microcusteio de baixo para cima como padrão-ouro para as avaliações econômicas em saúde, tendo em vista que a coleta dos dados individuais possibilita o maior nível de precisão na estimativa dos custos (Drummond et al., 2005; Hrifach et al., 2016; Tan, 2009). Considerando essa posição e retomando o objetivo de esclarecer as recomendações sobre estudos de custos em saúde, este artigo detalha as ferramentas, vantagens e desafios de conduzir os estudos de Microcusteio.

\section{Microcusteio}

Nos estudos de Microcusteio, todos os componentes de custo são definidos no nível mais detalhado, a partir de dados individuais do tratamento do paciente, por exemplo, da revisão do prontuário ou da ficha clínica do estudo. A unidade de análise em Microcusteio é o serviço individual, uma vez que, considerando a perspectiva da análise do estudo, o método procura avaliar os custos com a maior precisão possível, incluindo os custos diretos e indiretos dos cuidados prestados ao paciente.

A avaliação dos custos individuais dos cuidados de saúde auxilia a identificar se as diferenças de custo entre as opções terapêuticas em avaliação surgem da variação dos valores monetários dos recursos consumidos ao longo do processo de cuidado ou da intensidade da utilização desses recursos (Jackson, 2000). Entre as principais vantagens do Microcusteio, encontram-se (Tan, 2009): o alto nível de precisão em relação ao tratamento ou serviço avaliado, a partir do qual podem ser elaboradas políticas públicas específicas; e a possibilidade de agrupamento dos componentes de custo por subgrupos de pacientes, por diagnóstico, tratamento ou outros grupos de interesse, permitindo análises individualizadas (Tan, 2009)

Um desafio no desenvolvimento do Microcusteio é a viabilidade de se realizar esse tipo de estudo devido a diversos motivos, por exemplo, a falta de padronização de métodos para a coleta de dados de custos e as especificidades institucionais que dificultam a comparabilidade dos resultados obtidos (Donovan et al., 2014). Além disso, o processo de coleta de dados é demorado e oneroso e pode haver normativas éticas locais relacionadas à acessibilidade, à privacidade e à confidencialidade dos dados dos pacientes que restrinjam ou impossibilitem a coleta de dados (Frick, 2009; Jackson, 2000; Tan, 2009). É usualmente aceito que os estudos apresentem mais de uma abordagem, utilizando Microcusteio de baixo para cima para os componentes de custo que se acredita terem impacto no custo total e a abordagem de Microcusteio de cima para baixo para os demais componentes (Drummond et al., 2005; Tan, 2009).

Da mesma forma que na abordagem de baixo para cima, o Microcusteio de cima para baixo identifica todos os componentes de custos, porém os dados provêm de fontes mais abrangentes, por exemplo, as contas anuais, e, por meio de um processo de desagregação, chega-se ao nível do paciente. A abordagem de cima para baixo permite, então, o cálculo de custos para pacientes médios e inviabiliza o rastreamento dos custos aos pacientes individualmente e a realização de análises estatísticas de custos para detectar diferenças entre os pacientes (Clement Nee Shrive et al., 2009; Tan, 2009). Segundo Tan (Tan et al., 2012), os passos para a estimativa de custo baseado em Microcusteio seriam: definição da perspectiva de análise; definição da unidade de análise; identificação de itens de custo; mensuração dos itens de custo; valoração dos itens de custo; e avaliação de incertezas nas estimativas. Como formas de 
condução dos estudos, a literatura tem explorado métodos de gestão de custos contemporâneos, por exemplo, o método de custeio baseado em atividades e tempo (TDABC) (Kaplan \& Anderson, 2007; Keel et al., 2017).

\section{Método de custeio por atividade e tempo (TDABC) em estudos de Microcusteio}

Os métodos de custeio por atividade atribuem que múltiplos produtos consomem as mesmas atividades e recursos da estrutura da empresa em proporções diferentes (Martin et al., 2018). Recursos são elementos econômicos que são aplicados ou utilizados na performance das atividades, tais como salários, medicamentos e material médico-hospitalar. As atividades são um conjunto de ações realizadas por meio de uma organização, úteis para a proposta do ABC. Os objetos de custo são qualquer paciente, patologia, serviço, contrato, projeto ou outra unidade de trabalho cuja medida de custo é desejada (Souza, 2013). Ao se usar o TDABC, o custo de um produto ou serviço torna-se mais acurado, principalmente quando ele é formado por uma parcela de atividades indiretas como em um ambiente hospitalar

Os métodos de custeio baseados em atividades, ao contrário do método de custeio por absorção, calculam o custo do serviço prestado ao paciente a partir da referência de consumo de atividades e, consequentemente, recursos que cada serviço utiliza (bottom-up). Já o método por absorção distribui gastos aos departamentos e sequentemente aos serviços (top-down) (Erhun et al., 2015). A Figura 1 ilustra a comparação de como a informação custo é calculada no método por absorção e nos métodos de custeio por atividade.

A mecânica do método TDBC pode ser fragmentada em sete etapas: cálculo do custo de cada departamento; definição da capacidade padrão de cada departamento; cálculo da Taxa de Custo Unitário (TCU) de cada departamento; identificação das atividades desenvolvidas por cada departamento; estimativa do tempo de processamento de cada serviço; estruturação das equações de tempo; cálculo do custo dos serviços.
Em 2013, a Clínica Mayo, em parceria com Harvard Business School, começou a aplicar o método TDABC para mensurar o custo de três diferentes procedimentos: cirurgia ortopédica, medicina cardiovascular e cuidado em atenção primária (Haas et al., 2015). Entre as principais vantagens da aplicação do método, os autores destacam que foi possível identificar oportunidades de criação de valor a partir da eliminação e/ou redução de atividades que não agregam valor nesses procedimentos e, ao serem executadas, representam custos. A construção do método, por detalhar o processo em fluxo, também auxiliou a disseminação do conhecimento e identificação de oportunidades de como criar valor entre os funcionários (Haas et al., 2015). Para alcançar tais objetivos, definir a perspectiva e a unidade de análise para a qual o estudo está sendo realizado é fundamental, para que os métodos permitam alcançar os objetivos desejados (McBain et al., 2016).

\section{Perspectiva da análise de custos}

Em um estudo de custos, o objetivo do estudo está diretamente relacionado com a perspectiva do estudo, e ambos indicarão quais custos deverão ser mensurados. A perspectiva diz respeito ao ponto de vista pelo qual o problema é encarado e responde, em linhas gerais, quem é o pagador dos serviços descritos na análise (Drummond et al., 2015).

As perspectivas mais comumente utilizadas nos estudos de custos são aquelas relacionadas: i) à sociedade como um todo; ii) ao paciente; iii) ao sistema de saúde público ou suplementar; iv) ao prestador do serviço de saúde (Brasil, 2008; Brasil, 2013; Brasil, 2014; Hendriks et al., 2014). Porém, outras perspectivas são possíveis em cenários específicos, por exemplo, a de um empregador decidindo se deve prover vacinação contra a gripe para os funcionários ou a de uma operadora de saúde suplementar decidindo se deve oferecer um programa de reabilitação gratuito aos seus beneficiários.

Qualquer que seja a perspectiva do estudo, é fundamental que ela esteja declarada desde o início, pois será a determinante de quais custos serão considerados. Estudos de custos

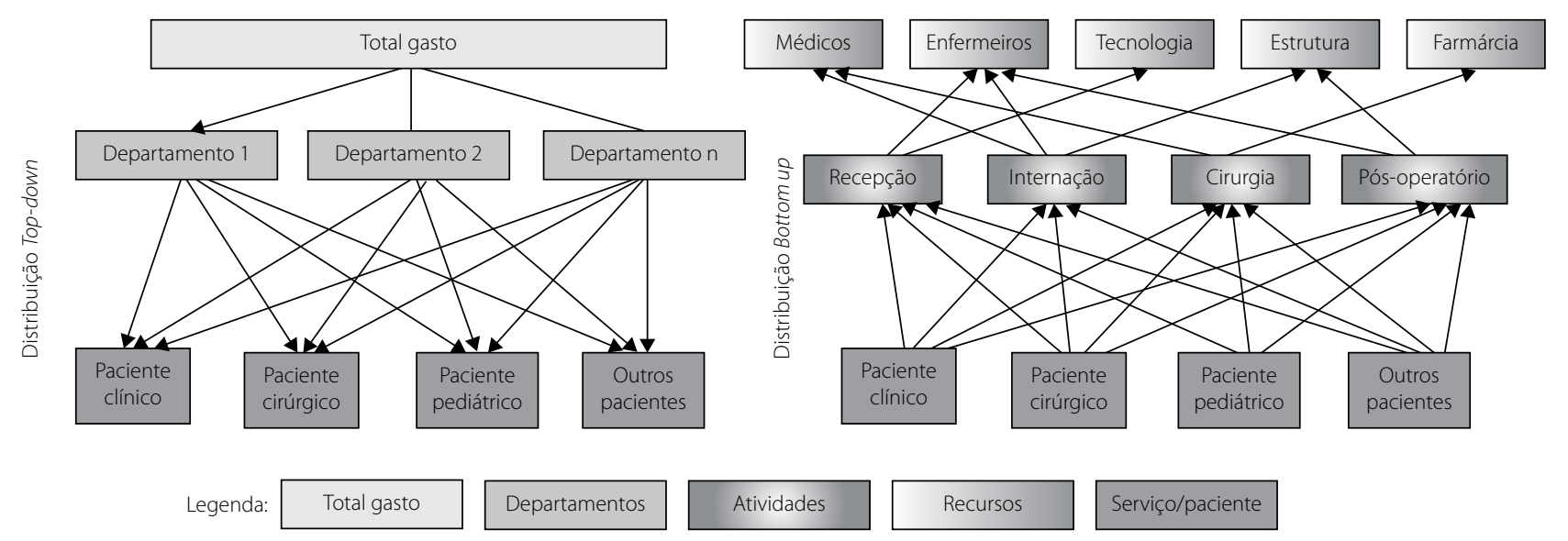

Figura 1. Comparação entre os métodos de custeio. 
podem ser realizados nos diferentes níveis da atenção à saúde e com diferentes níveis de abrangência, por exemplo, em Unidade Básica de Saúde ou hospital, e abrangendo toda a instituição ou somente parte dela, conforme o objetivo do estudo (Araujo, s/d.; Drummond et al., 2005; Hendriks et al., 2014; Oliveira et al., 2014).

Cabe destacar a dificuldade em definir custos do sistema público e os custos de um provedor público específico. No primeiro caso, trata-se da perspectiva do órgão gestor do SUS como comprador de serviços públicos e privados de saúde, e todos os custos econômicos diretos cobertos pelo sistema público devem ser computados, independentemente de qual provedor é a fonte do custo. Como o aferido deve refletir o custo dispendido pelo pagador final (sistema público), frequentemente são utilizadas tabelas de reembolso de procedimentos do SUS para essa aferição, que representariam o quanto o sistema paga por determinado item, independentemente do consumo real de recursos do prestador final. Por outro lado, na perspectiva do prestador final, a tabela de repasse representaria receita, não gastos, e assim seria inadequado utilizar esses valores. Nesse caso os gastos reais do prestador deveriam ser identificados nas folhas de pagamento, notas de compra e demais listagens administrativas disponíveis do local.

Porém, com a crescente utilização de contratos com orçamentos globais para prestadores públicos (particularmente hospitais), atualmente as tabelas de repasse do SUS não necessariamente refletem nem o efetivamente dispendido pelo gestor ou sistema, nem o efetivamente gasto pelo prestador. Nesse cenário, estudos de custos da perspectiva de prestadores públicos de referência (ou de prestadores privados dos quais o sistema público compra serviços) são provavelmente a melhor estimativa do custo real de uma tecnologia para o sistema, particularmente ao ser considerado que os gastos das esferas municipal, estadual e federal deveriam ser todos incluídos na perspectiva de Sistema Público (Figura 2).

Na perspectiva da sociedade, todos os custos econômicos diretos e indiretos são avaliados e incluídos. É considerada a perspectiva mais abrangente e recomendada por diversos autores, contudo nem sempre é viável em virtude das restrições de disponibilidade de dados (Araujo, s/d.; Drummond et al., 2005; Hendriks et al., 2014). Ainda, como na perspectiva da sociedade devem necessariamente ser incluídos os custos econômicos indiretos, é preciso cuidado para não realizar dupla contagem de custos quando da realização de estudos de custo-utilidade.

A Diretriz Metodológica de Avaliações Econômicas recomenda, para submissões de estudos às esferas públicas competentes, que a perspectiva escolhida para os estudos de avaliação econômica seja a do SUS como órgão comprador de serviços (ou seja, computando todos os custos cobertos pelo sistema público de saúde) (Brasil, 2012). Uma análise adicional recomendada é pela perspectiva da sociedade, na qual devem ser incluídos todos os custos da produção do serviço/procedimento e do tempo perdido pelos pacientes e seus familiares, além dos custos relacionados à perda de produtividade e morte prematura (Brasil, 2012).

\section{Unidade de análise}

A Unidade de Análise é o objeto de interesse para a identificação dos custos, tendo estreita relação com a tomada de decisão que a informação de custos vai subsidiar, considerando a perspectiva da análise do estudo. Assim como na perspectiva
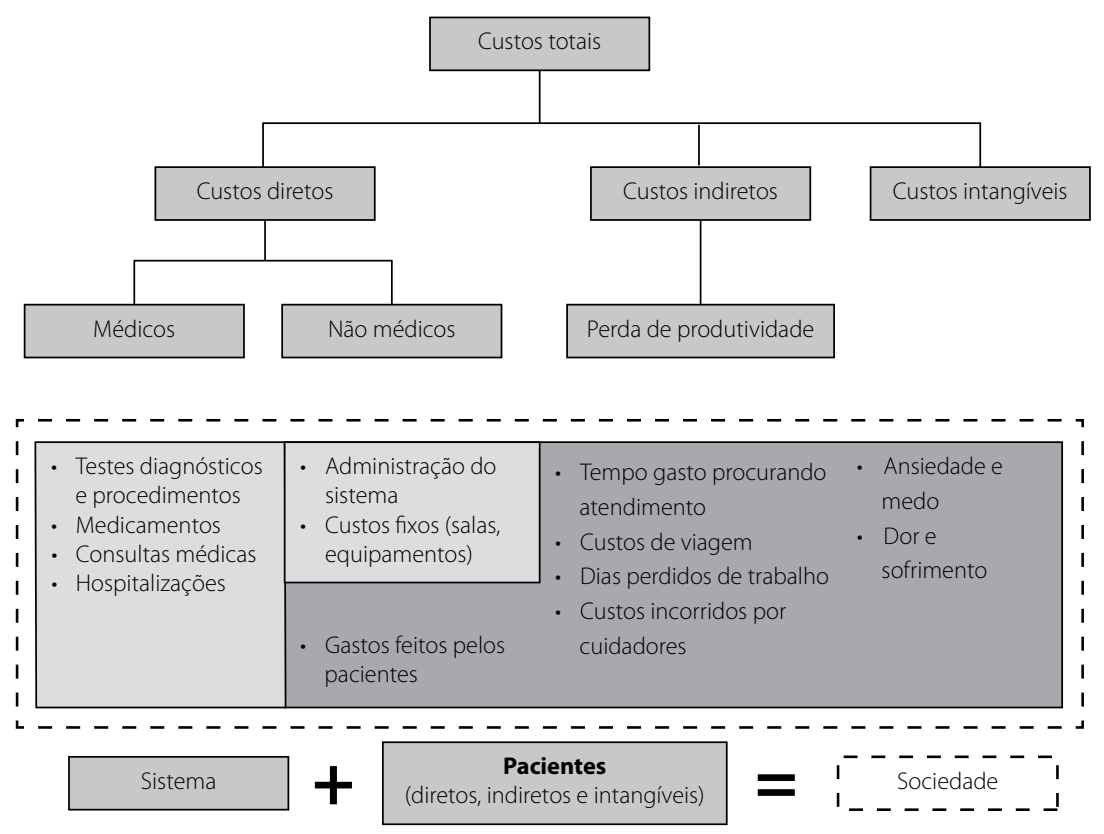

Figura 2. Categorias de custos em saúde quanto à alocação e quanto à perspectiva do estudo. 
do estudo, a definição da unidade de análise também depende do objetivo do estudo. Por exemplo, se o objetivo é avaliar se os preços pagos por um determinado convênio para a realização de uma angioplastia coronariana primária suportam os custos relativos ao procedimento, a unidade de análise deverá ser o procedimento (Hendriks et al., 2014).

$\mathrm{Na}$ condução de estudos de custos da doença ou de um estudo de custo para posterior Avaliação Econômica, o custeio de todo o processo assistencial (acolhimento, diagnóstico, tratamento, assistência em complicações e nas comorbidades) representa a aferição mais abrangente e, em geral, esperada para tomada de decisão. Porém, nem sempre é necessário aferição de toda cadeia assistencial, dependendo da questão de pesquisa a ser respondida. A definição da unidade de análise e sua associação com o processo decisório determinará o método de custeio ou a combinação mais adequada.

A produção de serviços em saúde envolve, fundamentalmente, profissionais de saúde, materiais, estrutura física e equipamentos, que genericamente podemos denominar de recursos. Em geral, cada atendimento de um paciente utiliza diversos recursos associados a múltiplos atendimentos, daí a dificuldade em apurar os custos de um atendimento específico e a necessidade de metodologias de custeio que sistematizem o processo de cálculo de custos como o TDABC apresentado. É importante que, uma vez definidas as perspectivas e a unidade de análise do estudo, todos os recursos correspondentes sejam considerados.

\section{Identificação dos itens de custos}

A identificação dos itens de custos que serão considerados na análise depende da perspectiva do estudo (Brasil, 2008; Oliveira et al., 2014). A Tabela 1 mostra quais custos devem ser incorporados na análise considerando as diferentes perspectivas.

Os itens de custos médicos diretos relativos a consultas, exames, procedimentos e internações podem ser identificados por meio da revisão do prontuário do paciente, coleta de dados prospectiva, observação direta, registro de atividades, relatórios administrativos ou de relatórios informatizados nos casos em que o hospital utiliza prontuário eletrônico (Frick, 2009). Para os itens de custos não médicos, as informações podem ser obtidas por meio de coleta de dados prospectiva com o paciente ou familiares e cuidadores por meio da aplicação de questionários estruturados ou do diário do paciente (Frick, 2009).

Um fator importante para a reprodutibilidade do estudo em outras instituições é a descrição detalhada de quais itens de custos serão incluídos na análise, os critérios de seleção desses itens e a fonte de coleta de dados.

\section{Mensuração e valoração dos itens de custo}

$\mathrm{Na}$ avaliação dos custos individuais, os custos são calculados por meio da multiplicação de preços pela quantidade de recursos (Drummond et al., 2005). Em análises econômicas, são usadas três formas distintas de aferição: i) uso de dados administrativos dos hospitais para verificar o preço de aquisição dos itens de custos; ii) utilização de fontes secundárias de preços, tais como tabelas de reembolso ou de fixação de preços de mercado; e iii) estimativas baseadas em informações da literatura. As formas ii e iii fogem ao escopo deste artigo e estão descritas na literatura da área (Drummond et al., 2005). A busca de dados primários para a valoração de recursos é discutida nas seções a seguir, com abordagem em separado, visto que diferentes componentes do custo trazem desafios distintos à sua valoração.

De forma geral, recomenda-se que a primeiras informações na valoração dos itens de custo sejam a moeda e o ano em que o estudo foi realizado. Também deve ser descrito o tipo de abordagem utilizada para valoração de cada item de custo: de baixo para cima, de cima para baixo ou mista (Hrifach et al., 2016; Tan et al., 2012). Dependendo da perspectiva do estudo, atenção especial deverá ser dada aos custos econômicos indiretos relativos à perda de produtividade, abordados em seção específica.

Tabela 1. Custos a serem considerados de acordo com a perspectiva do estudo (Oliveira et al., 2014)

\section{Perspectiva do Estudo}

\begin{tabular}{lllll}
\cline { 2 - 4 } Tipos de custos & Sociedade & Pacientes e familiares & $\begin{array}{l}\text { Setor público ou } \\
\text { hospitais sem fins } \\
\text { lucrativos }\end{array}$ & Seguradora de Saúde \\
\hline Tratamento médico & $\begin{array}{l}\text { Todos os custos do } \\
\text { tratamento }\end{array}$ & Despesas pessoais & $\begin{array}{l}\text { Pagamento dos serviços } \\
\text { utilizados }\end{array}$ & $\begin{array}{l}\text { Pagamento dos serviços } \\
\text { cobertos }\end{array}$ \\
\hline $\begin{array}{l}\text { Tempo do paciente com } \\
\text { o tratamento }\end{array}$ & $\begin{array}{l}\text { Custo de todo o tempo } \\
\text { utilizado }\end{array}$ & Custo de oportunidade & Nenhum & Nenhum \\
\hline $\begin{array}{l}\text { Tempo de familiares ou } \\
\text { terceiros }\end{array}$ & Todos os custos & Somente despesas diretas & Nenhum & Nenhum \\
\hline $\begin{array}{l}\text { Transporte e outros } \\
\text { serviços não médicos }\end{array}$ & Todos os custos & Todos os custos & Nenhum & Nenhum ou somente \\
\hline
\end{tabular}




\section{Equipe de saúde}

No Microcusteio de baixo para cima, os custos da equipe que presta diretamente os cuidados assistenciais são estimados para cada paciente, sendo necessários os dados de custos de pessoal, carga horária de trabalho e tempo para a realização do cuidado (Hendriks et al., 2014; Tan, 2009). Recomenda-se a descrição das categorias profissionais consideradas na análise.

Antes de iniciar a valoração dos custos da equipe, é necessário quantificar a carga de trabalho dos diversos profissionais envolvidos na assistência ao paciente. A carga de trabalho é determinada por meio da identificação das intervenções requeridas pelos pacientes e do tempo despendido pelos profissionais, na sua realização.

\section{Valoração de recursos}

$\mathrm{Na}$ etapa de valoração de recursos, os custos de pessoal devem incluir o salário e encargos legais e sociais definidos pela legislação, por exemplo, repouso semanal remunerado, férias e adicional, décimo terceiro salário, contribuição previdenciária, contribuição ao Fundo de Garantia por Tempo de Serviço (FGTS), abonos de faltas e direitos relativos às convenções coletivas de trabalho (Martins, 2010). Recomenda-se que na valoração dos custos da equipe seja informado o percentual aplicado para os encargos legais, sociais e benefícios, bem como a descrição dos encargos e benefícios considerados, de forma a viabilizar a reprodutibilidade e a comparação entre estudos. Na perspectiva do hospital, a informação desse percentual pode ser obtida no departamento de recursos humanos/ pessoal ou no departamento de controladoria da instituição.

A carga horária de trabalho é variável, conforme a categoria profissional e estabelecida no contrato do trabalhador, contudo, por definição legal, o teto máximo é de 44 horas semanais (Martins, 2010). A informação de carga horária pode ser obtida no departamento de recursos humanos ou na legislação vigente.

A estimativa do tempo utilizado no cuidado do paciente deve, preferencialmente, ser realizada por meio de estudo de tempos e movimentos, que consiste na observação direta das atividades realizadas pelo profissional e determinação do tempo médio padrão de execução de cada atividade (Hendriks et al., 2014; Chiavenato, 2004). Outra forma de coleta desses dados pode ser a utilização de questionários, registro autorreferenciado de atividades e entrevistas com a equipe (Hendriks et al., 2014). A determinação de custos da equipe na abordagem de Microcusteio de cima para baixo pode ser mais viável que a abordagem de baixo para cima, em virtude de requerer os dados no nível de departamento, porém avalia cada componente de custo para pacientes médios.

Estudo de Microcusteio de cima para baixo realizado na área de oncologia e hematologia em 30 hospitais nos Países Baixos estimou que, em consultas ambulatoriais, os custos com médicos especialistas representam entre 50\% e 69\% dos custos de pessoal direto. Na internação hospitalar, os custos de pessoal direto variaram de $45 \%$ a $59 \%$, e a equipe de enfermagem foi o maior responsável pelos custos de pessoal direto, com variações entre $25 \%$ e $67 \%$. Porém, o estudo não considerou custos de exames laboratoriais e de imagem, nem de medicamentos (Tan, 2009).

\section{Medicamentos e outros itens de consumo}

$\mathrm{Na}$ abordagem de Microcusteio de baixo para cima, a valoração dos custos de medicamentos e consumíveis deve ser realizada utilizando-se preços unitários por medicamento ou consumível por paciente. As fontes de dados podem vir de bases nacionais ou sistemas administrativos e contábeis do hospital (Clement Nee Shrive et al., 2009; Hendriks et al., 2014; Tan et al., 2012). O cálculo de custos pode ser obtido por meio da divisão do custo total do medicamento ou consumível pelo número total de pacientes (Hendriks et al., 2014).

Para medicamentos, nos estudos com coleta de dados dos custos diretos não médicos do paciente, a informação do preço e do local de aquisição pode não estar disponível. Nesses casos, o preço pode ser estabelecido por meio do preço médio obtido na farmácia popular ou na rede privada local. Nesta última, recomenda-se não utilizar o preço de oferta, uma vez que a oferta é momentânea e pode não ocorrer nas outras farmácias pesquisadas, gerando subestimação no preço médio. Não havendo medicamento genérico, recomenda-se a utilização da marca de menor preço (Ribeiro et al., 2005).

Drummond et al. recomendam o preço médio de medicamentos do atacado como representativo dos custos reais dispendidos pelo paciente e recomenda a utilização de média de preços para valoração dos custos não médicos dos medicamentos (Drummond et al., 2005).

\section{Estrutura física e custos operacionais}

Os custos de estruturas físicas são importantes no estudo de Microcusteio. No entanto, o processo de obtenção dessa informação é complexo devido à dificuldade de mensuração do valor unitário por paciente versus a taxa horária de uso, ou seja, o quanto cada paciente "usufruiu" das estruturas físicas durante seu tratamento. Os custos operacionais gerais, também conhecidos como overhead, referem-se a todos os outros gastos de produção utilizados para manter o local em funcionamento onde o serviço é realizado. Tipicamente são considerados custos operacionais água, eletricidade, seguros, limpeza e manutenção e capital (depreciação de construção e equipamentos) (Tan, 2009).

De acordo com o método escolhido para a aferição dos custos, a forma de obtenção dessa informação pode ser distinta. Considerando o TDABC como método de condução do estudo de Microcusteio e usufruindo das estruturas de rateio por departamentos que a controladoria do hospital elabora, podem-se usar as informações de custos dos departamentos, trabalhando-se nos dados que representam custos fixos de estrutura 
(Kaplan, 2014). Por exemplo, é comum no rateio por absorção do hospital incluir a folha de pagamento dos profissionais médicos e de enfermagem de cada departamento. Esse dado, em um estudo de Microcusteio, é trabalhado de forma separada, como custos de equipe ou profissionais. Dados que compõem o custo da estrutura hospitalar como depreciações, aluguéis e energia, alocados aos departamentos que o paciente consome, podem ser utilizados considerando as atuais distribuições de custos feitas pelo departamento de controladoria da instituição.

Assim, para obter o custo da estrutura por unidade de tempo de um departamento, deve ser avaliada a distribuição de custos fixos operacionais desse departamento e, na sequência, dividir o custo fixo médio do departamento pela sua capacidade horária de produção. Por exemplo, em uma unidade de internação que possui 20 leitos, a capacidade de atendimento horário equivale a 20 leitos, abertos 24 horas por dia ao longo de 30 dias por mês, ou 14.400 horas mensais. Se essa unidade de internação tem contas fixas médias de $\mathrm{R} \$ 140.400,00$ por mês, o custo por unidade de hora dessa unidade de internação equivale a $\mathrm{R} \$ 10,00$. Esse levantamento deve ser feito para todos os departamentos que o paciente em análise consome ao longo da sua internação.

O Programa Nacional de Gestão de Custos em Saúde, com o intuito de auxiliar no processo de apuração e gestão de custos em unidades de Saúde do SUS, de forma padronizada e estruturada, vem implantando o Sistema de Apuração e Gestão de Custos do SUS (APURASUS). No APURASUS são definidos critérios de rateio para a alocação de custos nos hospitais SUS do Brasil.

\section{Considerações finais}

A contextualização dos estudos de custos em saúde sobre a perspectiva das diferentes áreas de pesquisa e operação do serviço de saúde, que utilizam a informação custos, tem o propósito de contribuir com o avanço da acurácia da apropriação desses dados no país e homogeneizar a comunicação entre estudos conduzidos por diferentes escolas de pesquisa (econômica, contábil, engenharia, saúde etc.). Este artigo sugere recomendações para a realização de estimativas de custos em saúde, utilizando a metodologia de Microcusteio, considerada como padrão-ouro para a identificação dos custos em saúde. Os métodos de definição do estudo, coleta e análise de dados apresentados passam a ser recomendáveis às avaliações de custos de saúde, principalmente para o uso da informação custo em avaliações econômicas de saúde.

\section{Referências bibliográficas}

Araujo DV. Temas Importantes na Saúde: custos e gerenciamento de doenças. s/d. Available from: http://www.iats.com.br/eng/download/Temas_ Importantes_na_Sa\%FAde.pdf.

Brasil. Ministério da Saúde. Avaliação econômica em saúde: desafios para gestão no Sistema Único de Saúde. 1ª ed. Série A. Normas e Manuais Técnicos. Brasília, DF: Editora do Ministério da Saúde; 2008.
Brasil. Ministério da Saúde. Secretaria de Ciência, Tecnologia e Insumos Estratégicos. Departamento de Ciência e Tecnologia. Diretrizes Metodológicas: Diretriz de Avaliação Econômica. 2a ed. Brasília, DF: Ministério da Saúde; 2012. 76p. (Série A: Normas e Manuais Técnicos)

Brasil. Ministério da Saúde. Secretaria de Ciência, Tecnologia e Insumos Estratégicos. Departamento de Ciência e Tecnologia. Diretrizes Metodológicas. Estudos de Avaliação Econômica em Saúde. 2a ed. Série A. Normas e Manuais Técnicos. Brasília, DF: Ministério da Saúde; 2014.

Brasil. Ministério da Saúde. Secretaria-Executiva. Departamento de Economia da Saúde, Investimentos e Desenvolvimento. Glossário Temático. Economia da Saúde, 3a ed. 1a reimpressão. Brasília, DF: Ministério da Saúde; 2013.

Brasil. Presidência da República. Casa Civil. Subchefia para Assuntos Jurídicos. Lei no 12.401, de 28 de abril de 2011.

Chiavenato I. Introdução a Teoria Geral da Administração Compacta. $3^{a}$ ed. Rio de Janeiro, RJ: Campus; 2004.

Clement Nee Shrive FM, Ghali WA, Donaldson C, Manns BJ. The impact of using different costing methods on the results of an economic evaluation of cardiac care: microcosting vs gross-costing approaches. Health Econ. 2009;18(4):377-88.

Donovan CJ, Hopkins M, Kimmel BM, Koberna S, Montie CA. How Cleveland Clinic used TDABC to improve value. Healthc Financ Manage. 2014;68(6):84-8.

Drummond MF, Sculpher MJ, Claxton K, Stoddart GL, Torrance GW. Methods for the economic evaluation of health care programmes. Oxford: Oxford University Press; 2015.

Drummond MF, Sculpher MJ, Torrance GW, O'Brien BJ, Stoddart GL. Methods for the Economic Evaluation of Health Care Programmes. 3rd ed. Oxford: Oxford University Press; 2005.

Erhun F, Mistry B, Platchek T, Milstein A, Narayanan VG, Kaplan RS. Time-driven activity-based costing of multivessel coronary artery bypass grafting across national boundaries to identify improvement opportunities: study protocol. BMJ Open. 2015.

Frick KD. Microcosting quantity data collection methods. Med Care. 2009;47(7 Suppl 1):S76-81.

Haas DA, Helmers RA, Rucci M, Brady M, Kaplan RS. The Mayo Clinic Model for Running a Value-Improvement Program. 2015. Available from: https://hbr. org/2015/10/the-mayo-clinic-model-for-running-a-value-improvementprogram.

Hendriks ME, Kundu P, Boers AC, Bolarinwa OA, Te Pas MJ, Akande TM, et al. Step-by-step guideline for disease-specific costing studies in low- and middle-income countries: a mixed methodology. Glob Health Action. 2014;7:23573.

Hrifach A, Brault C, Couray-Targe S, Badet L, Guerre P, Ganne C, et al. Mixed method versus full top-down microcosting for organ recovery cost assessment in a French hospital group. Health Econ Rev. 2016;6:53.

Jackson T. Cost estimates for hospital inpatient care in Australia: evaluation of alternative sources. Aust N Z J Public Health. 2000;24(3):234-41.

Kaplan RS. Improving value with TDABC. Healthc Financ Manage. 2014;68(6):76-83.

Kaplan RS, Anderson SR. Time-driven activity-based costing: a simpler and more powerful path to higher profits. Boston: Harvard Business School Publishing Corporation; 2007.

Keel G, Savage C, Rafiq M, Mazzocato P. Time-driven activity-based costing in health care: A systematic review of the literature. Health Policy. Health Policy. 2017;121(7):755-63.

Kolaczinski J, Hanson K. Costing the distribution of insecticide-treated nets: a review of cost and cost-effectiveness studies to provide guidance on standardization of costing methodology. Malar J. 2006;5:37. 
Martin JA, Mayhew CR, Morris AJ, Bader AM, Tsai MH, Urman RD. Using TimeDriven Activity-Based Costing as a Key Component of the Value Platform: A Pilot Analysis of Colonoscopy, Aortic Valve Replacement and Carpal Tunnel Release Procedures. J Clin Med Res. 2018;10(4):314-20.

Martins E. Contabilidade de custos. 10ª ed. São Paulo: Atlas; 2010.

McBain RK, Jerome G, Warsh J, Browning M, Mistry B, Faure PAl, et al. Rethinking the cost of healthcare in low-resource settings: the value of time-driven activity-based costing. BMJ Glob Health. 2016;1:e000134.

Oliveira ML, Santos LMP, Silva EN. Methodological foundations for cost-ofillness studies in Brazil. Rev Nutr. 2014;27(5):585-95.

Piola SF, Vianna SM, orgs. Estado de uma nação: textos de apoio - Saúde no Brasil: algumas questões sobre o Sistema Único de Saúde (SUS). Brasília, DF: Instituto de Pesquisa Econômica Aplicada (Ipea); 2009.
Ribeiro RA, Mello RGB, Melchior R, Dill JC, Hohmann CB, Lucchese AM, et al. Annual cost of ischemic heart disease in Brazil: public and private perspective. Arq Bras Cardiol. 2005;85(1):3-8.

Souza AA. Gestão financeira e de custos em hospitais. São Paulo: Atlas; 2013.

Tan SS [tese]. Microcosting in economic evaluations: Issues of accuracy, feasibility, consistency and generalisability. Rotterdam: Erasmus Universiteit Rotterdam; 2009.

Tan SS, Bakker J, Hoogendoorn ME, Kapila A, Martin J, Pezzi A, et al. Direct cost analysis of intensive care unit stay in four European countries: applying a standardized costing methodology. Value Health. 2012;15(1):81-6. 